\title{
Feature Analysis of Kidney Ultrasound Image in Four Different Ultrasound using Gray Level Co-occurrence Matrix (GLCM) and Intensity Histogram (IH)
}

\author{
Nurul Aimi Shaharuddin', Wan Mahani Hafizah Wan Mahmud ${ }^{1}$
}

${ }^{1}$ Department of Electronic Engineering, Faculty of Electrical and Electronic,
Universiti Tun Hussein Onn Malaysia, Batu Pahat, Johor, Malaysia.

Received 10 January 2018; accepted 30 April 2018, available online 2 July 2018

\begin{abstract}
Misinterpretation analysis of ultrasound images has been huge issues in the world nowadays. Lack of skills and knowledge, as well as unclear ultrasound image due to the presence of speckle noise in ultrasound, are some factors lead to this issue. In this research, we compare 188 kidney ultrasound images from four different types of ultrasound machines, named as ultrasound A, B, C and D. Image pre-processing of images which involve cropping, enhancement, and filtering are performed before manual segmentation and texture analysis process to indicates the wanted region and improve contrast in each image. Texture analysis is performed using gray level co-occurrence matrix (GLCM) and intensity histogram (IH) to find differences and similarities in kidney image texture between all ultrasounds. Four GLCM parameters, contrast, correlation, energy and homogeneity and four parameters from IH (mean, standard deviation, variance, and skewness) used to indicate the most significant features between all ultrasound machines. Results show that contrast in GLCM is the most significant features that can be extracted from all four ultrasound machines and will be used in the classification process.
\end{abstract}

Keywords: Ultrasound, kidney, using gray level co-occurrence matrix, intensity histogram, texture analysis

\section{Introduction}

Kidney is a crucial organ in excretory system. It getting rid wastes and water from the blood, balance chemicals in the body, produce vitamin $\mathrm{K}$ and assist in controlling blood pressure. Hence, failure in the functioning of kidney system gives huge impact in one's life. Due to increasing cost of medical treatment nowadays, detecting of kidney failure from an early stage are more practical and benefit patients [1].

In order to detect kidney abnormalities, ultrasound is the most preferred medical modality compared to MRI and CT scan. It is non-invasive and does not expose the patient to any radiation [2]. Basically, there are four main types of ultrasounds in the world. There are two-dimensional ultrasound (2-D US), three-dimensional ultrasound (3-D US), four-dimensional ultrasound (4-D US), and doppler ultrasound. In analyse kidney's condition, most critical measurement is the longitudinal length. Hence, in our research, we are focusing on the 2-D US as it is a standard medical modality to be used by medical practitioners.

Due to the evolution in medical technology, researcher predicts that the number of ultrasound will increase with time. According to [3], in 2007, there were at least 200,000 low cost ultrasound machines in the world. However, in the researcher survey, most low cost ultrasound machines are a deficiency in terms of safety and output images [3]. To make it worse, the analysis of ultrasound images totally depends on knowledge and experiences of sonographer and physicians. Consequently, the possibility of human error during analysis process are high where it threaten patient condition.

The main reason of misdiagnosing is the medical practitioners fail to differentiate texture in ultrasound images due to the low quality of ultrasound image caused by speckle noise. To overcome this problem, we investigate the differences and similarities feature of kidney ultrasound images between low cost ultrasound machine and conventional ultrasound in four different twodimensional ultrasound machines.

\section{Literature review}

Texture analysis is a crucial process in image processing to identify hidden data, reducing the error on image besides improving the classification results. It defines the image texture based on coarsens, granulation, regularity, randomness and smoothness of image texture. Tamilselvi \& Thangaraj categorize texture analysis in four types; structural, statistical, model-based and transform based [4] while Ranjitha M. and G. M. Nasira listed three types of texture feature which are the spectral approach, statistical approach and structural approach [5]. Based on our knowledge, most of the research in kidney ultrasound images applied the statistical approach in analysing image texture.

According to [4], two popular techniques in texture features are first and second order coefficients and structural coefficients. Raja et al. extracted features in kidney US images based on content descriptive multiple features [3], geometric moments feature [4] and regional gray-level distribution [5]. The results show that feature extraction from kidney US images based on those features is possible and they are highly effective in classifying the kidney diseases and disorders. Karthikeyini et al. used principal component analysis (PCA) method and their analysis shows that there exists an appreciable measure of relevance for weight vector in classifying kidney images 
[4], [6]. Those previous works however still have weaknesses due to accuracy and processing time. Hence, we use different types of feature extraction parameters based on intensity histogram features and Gray Level CoOccurrence Matrix (GLCM) features for extracting features.

In [6], the researcher applied 5 techniques on texture analysis. There is first order statistic (FOS), gray level cooccurrence matrix (GLCM), run-length matrix (RLM), moment invariants and Law's texture energy measures. The research concluded that combination of RLM and GLCM produced optimal results compare with another method. M. Vasantaha et. al. [7] used intensity histogram features and GLCM in differentiating features of the normal image, benign image and malignant image in a mammogram. GLCM used five features (energy, contrast, correlation, entropy, and homogeneity) which result in classified malignant and benign tumors in the liver. Researcher applies GLCM with four features, angular second moment, correlation, inverse difference moment and entropy using Xilinx FPGA in analysis image [8]. Another researcher analysed texture of kidney images using statistical value, range, standard deviation and entropy to generate automatic kidney detection in ultrasound images [9]. In this study, the researcher use four different types of kidney images, normal, bacterial infection, cystic disease and kidney stone from one ultrasound machine whilst our study analysed normal kidney images from four different types of ultrasound machines.

\section{Methodology}

Materials and method for each experiment will be explained briefly in this section. Fig. 1 shows the experimental chart starts with image acquisition, image pre-processing and feature extraction.

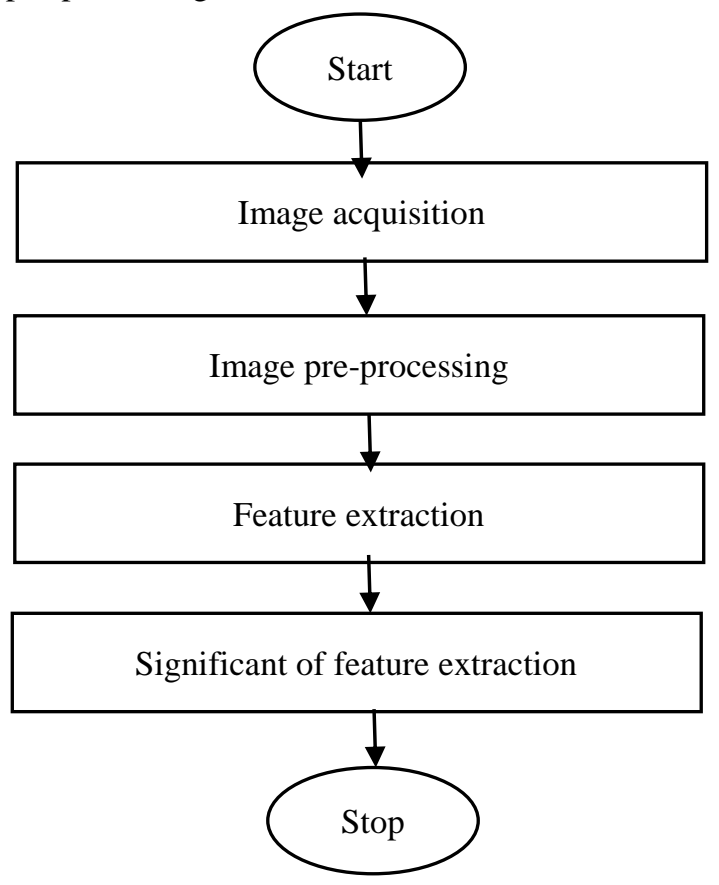

Fig.1 Experimental chart

\subsection{Image acquisition}

The subject involved in this research are healthy right kidney images from male and female kidney with age 25 to 35 years old. 47 kidney ultrasound images were extracted from four different ultrasound machines to analysed. We name each ultrasound as ultrasound A, B, C and $\mathrm{D}$. Ultrasound $\mathrm{A}$ is a portable ultrasound made in China. It costs RM7500. Ultrasound B is a conventional ultrasound with basic technical specifications while Ultrasound C and D are produced by top ultrasounds manufacturer in the world with 4-dimensional options provided in the machine. Compound imaging, tissue harmonics, speckle reduction, and storage file are four main technical specifications considered in these experiments. Table 1 indicates the technical specifications for those four ultrasound machines.

Table 1 Technical specifications for ultrasound

\begin{tabular}{ccccc}
\hline $\begin{array}{l}\text { Ultrasound } \\
\text { machine }\end{array}$ & $\mathrm{A}$ & $\mathrm{B}$ & $\mathrm{C}$ & $\mathrm{D}$ \\
\hline $\begin{array}{c}\text { Compound } \\
\text { imaging }\end{array}$ & $\mathrm{X}$ & $/$ & $/$ & $/$ \\
$\begin{array}{c}\text { Tissue } \\
\text { harmonics } \\
\begin{array}{c}\text { Speckle } \\
\text { reduction }\end{array}\end{array}$ & $\mathrm{X}$ & $/$ & $/$ & $/$ \\
Storage file & .bmp & .jpg & DICOM & DICOM \\
\hline
\end{tabular}

Compound imaging is a product of multiple frames from different angles or different frequency that combines to built a single multifrequency compound image [10]. It improves vision on low-contrast lesion besides used widely in the peripheral vasculature, breast and musculoskeletal system [11]. Compound imaging and tissue harmonics are two modes add in ultrasounds as a standard breast sonography and expected to improve detection and characterization of breast masses [10]

Tissue harmony is a situation where propagation of ultrasound beam through tissue generate higher harmonics frequencies which generate sonogram [12]. Tissue harmonics enhance lateral resolution, decrease side lobe artifacts, improved signal-to-noise ratio, and produce excellent contrast resolution [12], [13]. Plus, this leadingedge technology is a saviour on difficult-to-scan patients or patient with the obesity problem.

Speckle reduction is also known as speckle reduction imaging (SRI). It enhances the visibility of organs and lesions which improved contrast and decrease the impact of speckle noise.

Digital Imaging and Communications in Medicine (DICOM) standard, created by National Electrical Manufacturers Associations (NEMA) is the most standard for receiving medical image scans in hospital. DICOM is commonly used in medical storage. Research by Mr. D. C. Dhanwani and Prof. M. M. Bartere conclude that DICOM images produce more accurate result compare to nonmedical images (.jpg,.png,.bmp) [14]. On the other hand, BMP, introduced by Microsoft $\AA$ is supported by most 
applications. BMP is somewhat a simple format which lacks many features compared with others and it is said that BMP is the lowest common denominator format for interchanging images between programs [15]. Developed by PNG Development Group, PNG is a capable replacement for some uses of GIF and TIFF file. It includes metadata compression, built-in error and gamma correction and free compression algorithm [15].

\subsection{Image pre-processing}

Image pre-processing of kidney involved four steps, cropping the image, image enhancement, image filtering and image performance analysis. The flow chart in Fig. 2 displays the flow chart of the pre-processing kidney. Firstly, all the ultrasound images contain kidney will be cropped in rectangular size $400 \times 400$ pixels to remove unwanted part. Next, kidney image will be enhanced. For image enhancement, the contrast in the image will be increased. Since kidney image in ultrasound D has very low contrast, the ultrasound images were enhance using histogram equalization. Histogram equalization is a popular technique used by the previous researcher in enhanced ultrasound kidney images [16]-[19]. It will enhance the visibility of image as a whole which produces a uniform distribution of grayscale image [20]. Images then will be filtered using wiener filter with 3-by-3 window size.

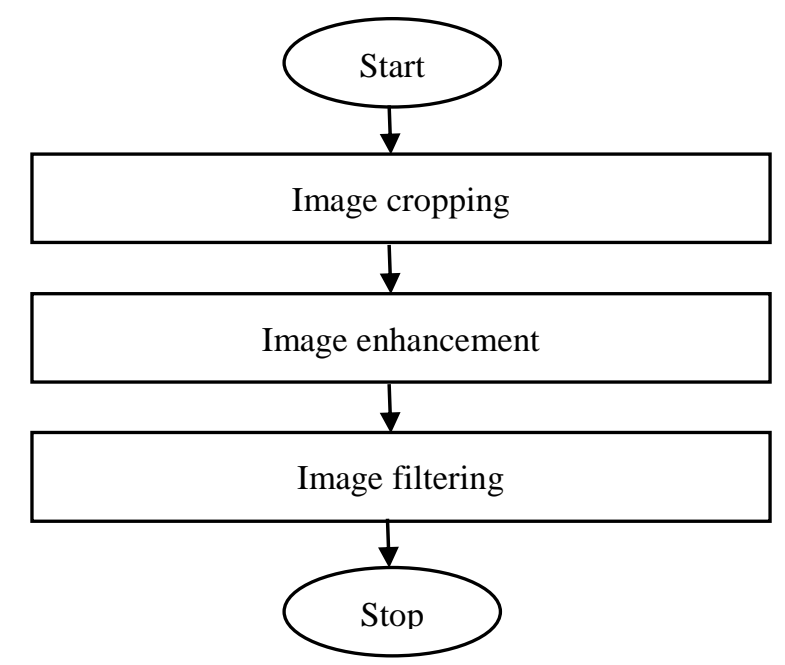

Fig. 2 Flow chart of pre-processing kidney

\subsection{Feature extraction}

Cropping images in 400x400 pixels contain kidney images and unwanted region. Therefore, manual cropping is done in each image. Next, two main popular methods, gray level co-occurrence matrix (GLCM) and intensity histogram (IH) used in to analyse the image. Main purposes of this step are to determine similar features that can be used by all four ultrasounds.

i. Gray level co-occurrence matrices (GLCM): Gray level co-occurrence matrices (GLCM), or also popular as gray tone spatial dependence matrix is a statistical method which calculates the rate of occurrence in a combination of pixel values in image spatial. The founder of GLCM, Haralick proposed 14 features to extracts from GLCM [21]. In GLCM, four parameters are used in achieving the objective of the experiment. There are contrast, correlation, energy, and homogeneity. The equation [22] of each feature is shown in equation (1) until (4);

Contrast $=\sum_{n=0}^{N_{g}-1} n^{2}\left\{\sum_{i=1}^{N_{g}} \sum_{j=1}^{N_{g}} p(i, j)\right\}$,

where $|i-j|=n$

Correlation $=\frac{\sum_{i} \sum_{j}(i, j) p(i, j)-\mu_{X} \mu_{y}}{\sigma_{X} \sigma_{y}}$

Energy $=\sum_{i} \sum_{j} p(i, j)^{2}$

Homogeneity $=\sum_{i} \sum_{j} \frac{1}{1+(i+j)^{2}} p(i, j)$

In equation (1) until $(4), p(i, j)$ is element of normalized co-occurrence matrix of filtered image from the previous step, $N_{g}$ is number of distinct gray level in quantized image, element $i$ and $\mathrm{j}$ indicates element row and column in image pixel while $\mu_{x} \mu_{y}$ are mean while $\sigma_{x} \sigma_{y}$ are standard deviation of marginal distribution of co-occurrence matrix.

ii. Intensity histogram (IH): In intensity histogram, mean, standard deviation, skewness and variance are four selected parameters. Intensity histogram analysis used widely in image processing. In this research, four features are considered; mean, standard deviation, skewness, and variance. Mean IH calculates the average of a pixel in the image and represents individual classification. Also known as the square root of the variance, standard deviation is a quantity measurement that calculates the area of deviation in a group and useful to characterize a cluster of the image. Meanwhile, skewness measure asymmetry of data around the sample means while variance is effective in determining the homogenous region of a pixel. Equation of each parameter are as in equation (5) until (8) [23];

Mean $=\frac{1}{T_{k}} \sum_{m} \sum_{\text {all }}^{n}[u(m-i, n-j)]$
Standard deviation $=$
$\sqrt{\frac{1}{T_{k}} \sum_{m} \sum_{\text {all }}^{n}[u(m-i, n-j)-M(i, j)]}$

Skewness $=$

$\frac{1}{T_{k}} \sum_{m} \sum_{\text {all }}^{n}[u(m-i, n-j)-M(i, j)]^{3}$ 
Variance $=$

$$
\frac{1}{T_{k}} \sum_{m} \sum_{\text {all }}^{n}[u(m-i, n-j)-M(i, j)]^{2}
$$

Where $u(m, n)$ is a discrete image, $T_{k}$ is sum of pixels in image pixels, $M$ is mean for image pixel $(i, j)$.

iii. Significant of texture analysis: In order to find the most relevant features that can be used in all ultrasounds, ANOVA technique is used. ANOVA is a technique in analysis of variance which determines differences between two or more independent groups. In our case, since our aim is to find the similarities feature of ultrasound, null hypothesis, $h_{o}$ with value higher than 0.05 will be choose to be used in the next step.

\section{Result and discussion}

For each ultrasound, the mean value for each features were calculated. Table 2 and 3 shows mean value of 47 kidney ultrasound images in GLCM features and IH for each ultrasound machine.

Table 2 Mean value of GLCM in ultrasound image

\begin{tabular}{lcccc} 
GLCM & \multicolumn{4}{c}{ Ultrasound machine } \\
feature & $\mathrm{A}$ & $\mathrm{B}$ & $\mathrm{C}$ & $\mathrm{D}$ \\
\hline Contrast & 0.06 & 0.06 & 0.06 & 0.06 \\
Correlation & 0.99 & 0.99 & 0.99 & 0.99 \\
Energy & 0.85 & 0.84 & 0.65 & 0.69 \\
Homogeneity & 0.99 & 0.99 & 0.987 & 0.99 \\
\hline
\end{tabular}

Table 3 Mean value of intensity histogram in ultrasound image

\begin{tabular}{lcccc}
\hline IH Feature & \multicolumn{4}{c}{ Ultrasound machine } \\
& $\mathrm{A}$ & $\mathrm{B}$ & $\mathrm{C}$ & $\mathrm{D}$ \\
\hline Mean & 24.03 & 24.54 & 55.66 & 34.04 \\
Standard & 61.45 & 58.97 & 67.21 & 52.09 \\
deviation & & & & \\
Skewness & 2.34 & 2.22 & 0.57 & 1.32 \\
Variance & 3788.06 & 3495.46 & 4562.90 & 2755.26 \\
\hline
\end{tabular}

Based on Table 2 and Fig. 3, ultrasound D has the highest value in contrast parameter while ultrasound B has the lowest value of contrast parameter. In correlation parameter, ultrasound $\mathrm{C}$ has the highest value while ultrasound $\mathrm{B}$ is the lowest. For energy parameter in GLCM, ultrasound $A$ has the highest value while ultrasound $\mathrm{C}$ has the lowest. Homogeneity, on the other hand, indicates the highest value for ultrasound $\mathrm{A}$ while ultrasound $\mathrm{C}$ is the lowest. For percentage difference in GLCM features, energy has the biggest difference between ultrasound machine $(26.60 \%)$ followed by contrast (9.39\%), homogeneity (1.21\%) and correlation (0.48\%).

From Table 3 and Fig. 4, in overall, features in intensity histogram produce a huge difference between one another. Ultrasound A and B produce almost similar value of intensity histogram features while ultrasound $\mathrm{C}$ has the lowest value in skewness but the highest in mean, standard deviation and variance feature. Ultrasound D generate the lowest value in standard deviation and variance.

Percentage difference in mean feature is $79.39 \%$ with the highest mean value is ultrasound $\mathrm{C}$ and lowest is ultrasound A. Standard deviation $(25.34 \%)$ has the lowest percentage difference between all ultrasound features. Ultrasound $\mathrm{D}$ indicates the lowest standard deviation value (52.09) while ultrasound $C$ is the highest (67.21). Variance feature also shows high difference value between all ultrasounds $(49.40 \%)$ with the highest value is ultrasound C (4562.90) and the lowest value is ultrasound D (2755.26). From the value of standard deviation and variance, it can be concluded that brightness of ultrasound images is as below:

Ultrasound $\mathrm{C}>$ ultrasound $\mathrm{A}>$ ultrasound $\mathrm{B}>$ ultrasound D

Ultrasound C produces skewness value less than 0.5 , that shows that image distribution in ultrasound image $\mathrm{C}$ is approximately symmetric. On the other hand, images in ultrasound A, B, and D are highly skewed as its value are more than 1. Skewness produces highest percentage difference between all the features $(121.60 \%)$.

Feature with small percentage difference between each ultrasound machine group then selected to be analysed with ANOVA using SPSS. For GLCM group, homogeneity, correlation, and contrast were selected while in intensity histogram parameter, the standard deviation is selected.

2

0
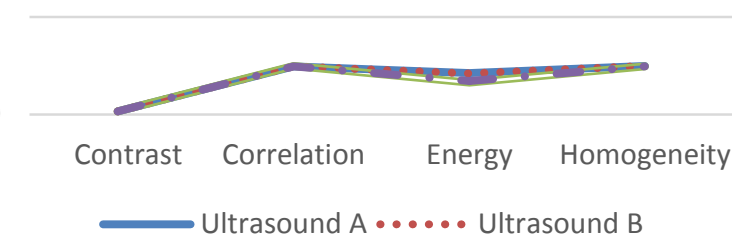

Ultrasound C - • Ultrasound D

Figure 3 Graph of mean value of GLCM in kidney ultrasound image

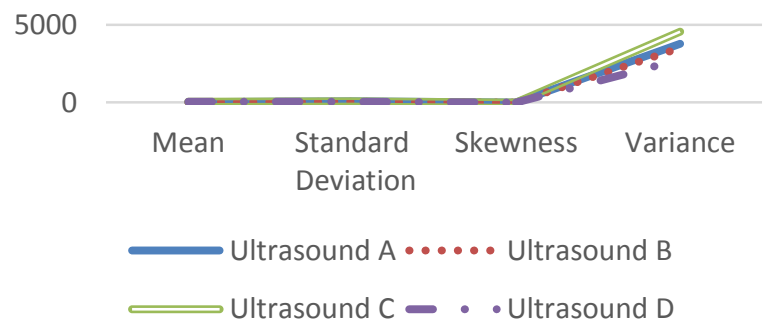

Figure 4. Graph of mean value of intensity histogram in kidney ultrasound image

Table 4 indicates ANOVA significance value between all ultrasound groups. The significant value of GLCM contrast is 0.09 , which is small than 0.05 . Hence, $h_{o}$ for GLCM contrast is rejected. Value of significance ANOVA for other parameters, GLCM correlation, GLCM 
homogeneity, IH standard deviation is 0 , which means that $h_{o}$ for these parameters is accepted. Since our experiment indicates similarities features between ultrasound, GLCM contrast will be used in the classification step as it rejected $h_{o}$.

Table 4 ANOVA significance value between all ultrasound groups

\begin{tabular}{ccc}
\hline Texture feature & Parameter & Value \\
\hline Gray level co- & Contrast & 0.09 \\
occurrence matrix & Correlation & 0 \\
(GLCM) & Homogeneity & 0 \\
Intensity Histogram & Standard & 0 \\
$(\mathrm{IH})$ & Deviation & \\
\hline
\end{tabular}

\section{Conclusions}

The output of ultrasound images is different from one ultrasound to another. 188 kidney ultrasound images from 4 different ultrasound machines have successfully extract. The result shows that GLCM energy and IH variance in four ultrasounds have huge different between groups. In terms of image brightness, ultrasound $\mathrm{C}$ produce the brightest image while ultrasound D is the darkest. Based on ANOVA test, GLCM contrast parameter has been choose to be used in the classification process.

\section{Acknowledgments}

This paper was partly sponsored by Center for Graduate Studies and Geran Penyelidikan Pascasiswazah (GPPS) grant no. U586.

\section{References}

[1] Ho, C., Pai, T., Peng, Y., Lee, C., Chen, Y., Chen, Y., and Chen, K. Ultrasonography image analysis for detection and classification of chronic kidney disease. Sixth International Conference on Complex, Intelligent, and Software Intensive Systems, (2012), pp. 624-629.

[2] Gheissari, A. The place of ultrasound in renal medicine. Saudi Journal of Kidney Diseases and Transplantation, Volume 17, (2006), pp. 540-548.

[3] Supriyanto, E. Development of performance and safety monitoring system for low cost ultrasound medical devices for prenatal diagnosis: Research project. (2008), pp. 75.

[4] Tamilselvi, M. P. R. and Thangaraj, D. P. Computer aided diagnosis system for stone detection and early detection of kidney stones. Journal of Computer Sceience, Volume 7, (2011), pp. 250-254.

[5] Ranjitha, M. and Nasira, G. M. Identifying renal calculi using different techniques. International Journal of Computer Applications, Volume 0975, (2015), pp. 15-18.

[6] Subramanya, M. B., Kumar, V., Mukherjee, S. and Saini, M. Classification of normal and medical renal disease using B-mode ultrasound images. Second
International Conference on Computing for Sustainable Global Development, (2015), pp. 19141918.

[7] Vasantha, M., Bharathi, D. V. S. and Dhamodharan, R. Medical image feature, extraction, selection and classification. International Journal of Engineering Science and Technology, Volume 2, (2010), pp. 20712076.

[8] Mohanaiah, P., Sathyanarayana, P. and Gurukumar, L. Image texture feature extraction using GLCM approach. International Journal of Scientific and Research Publication, Volume 3, (2013), pp. 1-5.

[9] Hafizah, W. M. and Supriyanto, E. Automatic generation of region of interest for kidney ultrasound images using texture analysis. International Journal of Biology and Biomedical Engineering, Volume 6, (2012), pp. 26-34.

[10] Mesurolle, B., Helou, T., El-khoury, M., Edwardes, M., Sutton, E. J. and Kao, E. Tissue harmonic imaging, frequency compound imaging, and conventional imaging. Ultrasound, Volume 26, (2007), pp. 1041-1051.

[11] Harvey, C. J., Pilcher, J. M., Eckersley, R. J., Blomley, M. J. K. and Cosgrove, D. O. Advances in ultrasound. Clinical Radiology, Volume 57,(2002), pp. 17551759.

[12] Shapiro, R. S., Wagreich, J., Parsons, R. B., StancatoPasik, A., Yeh, H. C. and Lao, R. Tissue harmonic imaging sonography: evaluation of image quality compared with conventional sonography. American Journal of Roentgenology. Volume 171, (1998), pp. 1203-1206.

[13] Ward, B., Baker, A. C. and Humphrey, V. F. Nonlinear propagation applied to the improvement of resolution in diagnostic medical ultrasound. Acoustical Society of America, Volume 101, (1997), pp. 143-154.

[14]Dhanwani , D. C. and Bartere, P. M. M. Survey on various techniques of brain tumor detection from MRI images. International Journal of Computational Engineering Research, Volume 4, (2014), pp. 24-26.

[15]Tan, L. K. Image file formats. Biomedical Imaging and Intervention Journal, Volume 2, (2006), pp. 1-7

[16] Raja, R. A. and Ranjani, J. J. Segment based detection and quantification of kidney stones and its symmetric analysis using texture properties based on logical operators with ultrasound scanning, International Journal of Computer Applications, (2013), pp. 8-15.

[17] Hafizah, W. M. and Supriyanto, E. Comparative evaluation of ultrasound kidney image enhancement techniques. International Journal of Computer Applications, Volume 21, (2011), pp. 15-19.

[18] Viswanath, K. and Gunasundari, R. Design and analysis performance of kidney stone detection from ultrasound image by level set segmentation and ANN classification. International Conference on Advances in Computing, Communications and Informatics, (2014), pp. 407-414.

[19]Jose, S. J., Sivakami, R., Maheswari, N. U. and Venkatesh, R. An efficient diagnosis of kidney images 
using association rules. International Journal of Computer Technology and Electronics Engineering, Volume 2, (2012), pp. 14-20.

[20]Zhang, X.-Y., Ge, L. and Wang, T.-F. Entropy-based local histogram equalization for medical ultrasound image enhancement. Second International Conference on Bioinformatics and Biomedical Engineering, (2008), pp. 2427-2429.

[21] Haralick, R. M. Statistical and structural approaches to texture. Proceeding of the IEEE, Volume 67, (1979), pp. 786-804.

[22] Khairuddin, W. N. H. B. W. Investigation of kidney ultrasound image texture features for healthy subject.
Universiti Tun Hussein Onn Malaysia, (2016).

[23] Hafizah, W. M., Supriyanto, E. and Yunus, J. Feature extraction of kidney ultrasound images based on intensity histogram and gray level co-occurrence matrix. Proceedings of the sith Asia International Conference on Mathematical Modelling and Computer Simulation, (2012), pp. 115-120. 